\title{
Seguro privado de saúde em Portugal: que evolução? Análise comparativa dos Inquéritos Nacionais de Saúde de 1995/1996 e de 1998/1999
}

\author{
Private health insurance in Portugal. \\ A comparative analysis of the National Health \\ Surveys, 1995-1996 and 1998-1999
}

Maria do Rosário Giraldes 1

1 Escola Nacional de Saúde Pública, Universidade Nova de Lisboa. Av. Padre Cruz 1600-560, Lisboa, Portugal.

\begin{abstract}
The National Health Survey (NHS) has been used to calculate the percentage of the population with private health insurance at both the nationwide and regional levels. Schooling and occupation have been used as proxies for income level in calculating the percentage of the population with private health insurance. The impact of chronic diseases has also been analyzed in relation to the purchase of private health insurance. A comparative analysis of the NHS from 1995-1996 to 1998-1999 showed an increase of 1\% and 0.7\% (for males and females, respectively) in the proportion of the population with health insurance. Level of income shows a clear influence on the acquisition of private health insurance. Individuals with medium and high levels of schooling, both males and females, are far more likely to have private insurance. Chronic diseases also have an impact on the purchase of private health insurance. According to regional analysis of NHS 1998-1999, the Greater Lisbon Metropolitan Area showed a higher percentage than the nation as a whole.
\end{abstract}

Key words Health Insurance; Social Class; Chronic Disease

Resumo Utilizou-se o Inquérito Nacional de Saúde (INS) para o cálculo da porcentagem de população com seguro privado de saúde, conforme o sexo, a faixa etária e o tipo de cobertura, em relação à população pesquisada em Portugal, na porção continental e em âmbito regional. Para o cálculo da população com seguro privado de saúde conforme o nível de renda, utilizou-se o grau de escolaridade e a profissão como indicadores indiretos. Além disso, procedeu-se à análise dessa população de acordo com a existência ou não de doenças crônicas. A análise comparada do INS 1995/1996 e INS 1998/1999 demonstra um crescimento, em três anos, respectivamente de $1 \%$ e 0,7\%, na porcentagem de população com seguro-saúde em relação à população pesquisada dos sexos masculino e feminino. Existe, ainda, uma clara associação do nível de renda com a procura por seguros-saúde. Os especialistas das profissões intelectuais são os que mais adquirem seguros privados de saúde. Quanto ao grau de escolaridade, a população com ensino médio ou superior é, de longe, a que apresenta as porcentagens mais elevadas. As doenças crônicas afetam a aquisição de seguros privados de saúde. A análise regional do INS 1998/1999 evidencia que a região de Lisboa e Vale do Tejo apresenta uma porcentagem superior à média da porção continental de Portugal.

Palavras-chave Seguro Saúde; Classe Social; Doença Crônica 


\section{Introdução}

O mercado de seguros privados de saúde varia consoante a importância do setor público de saúde no sistema de saúde de cada país. Um sistema de saúde misto, com um setor público de dimensão média, alcança melhores resultados em termos de bem-estar social, desde que haja uma boa distribuição de renda na população desse país (Marchand \& Schroyen, 2001).

Para Christiansen et al. (2001), a distribuição de renda e o grau de escolaridade desempenham um papel importante na aquisição de seguros privados de saúde. A probabilidade de ter seguro-saúde aumenta com a renda, a escolaridade obrigatória e os anos adicionais de freqüência de outros tipos de ensino. Também Propper (2000) conclui que o nível de renda afeta a decisão de adquirir seguros privados de saúde.

Além da distribuição de renda, também a incidência da doença ou a sua cronicidade desempenham um papel importante no mercado de seguros privados de saúde, uma vez que determinam o tipo de cobertura (Feldman et al., 1998; Neudeck \& Podezeck, 1996).

Em países que tradicionalmente possuem um serviço nacional de saúde, como o Reino Unido, tem-se verificado um aumento do setor privado, que vem a se refletir na aquisição de seguros privados de saúde, sobretudo para coberturas específicas, como cuidados de saúde dentários ou internação (Propper, 2000).

O sistema de saúde português caracterizase pela existência de um serviço nacional de saúde (SNS) de qualidade relativamente boa, que cobre a totalidade da população (cerca de 10 milhões de habitantes), apesar das longas listas de espera existentes em algumas especialidades. O SNS apresenta-se bem preparado para enfrentar o novo milênio, dado que possui um número relativamente baixo de leitos per capita (3,6 leitos por 1.000 habitantes), mais baixo do que o valor médio da União Européia, de 5,1 leitos por 1.000 habitantes, compatível com a noção de hospital do futuro. Essa noção implica uma completa reconceitualização, em que os grandes hospitais universitários serão substituídos por pequenas unidades de mais fácil gestão, administradas pelas autarquias ou por instituições com ou sem fins lucrativos e reforçadas por novas estruturas de apoio e bons serviços de assistência domiciliar.

O SNS português é igualmente caracterizado por uma boa rede de cuidados primários de saúde, embora ainda se deva melhorar a qualidade dos serviços prestados. Especial atenção deverá ser dada ao trade-off entre cuidados pri- mários de saúde e hospitalares, na medida em que muitas das urgências hospitalares podem ser consideradas como falsas urgências.

Pode-se considerar razoável a porcentagem de despesa total em saúde do PIB, de cerca de $9 \%$ no ano 2000. A questão principal nesse domínio é a elevada despesa privada em saúde não reembolsável, de cerca de $50 \%$ naquele ano, o que representa um grande esforço out-ofpocket por parte do consumidor de serviços de saúde (Giraldes, no prelo).

A existência de uma cobertura universal não impede, porém, que paralelamente venha a se desenvolver um mercado de oferta de seguros privados de saúde hoje disponíveis na maior parte das seguradoras do país.

Com efeito, o seguro privado de saúde, introduzido ainda há relativamente pouco tempo em Portugal, vem sendo adotado de forma a reforçar a cobertura do sistema básico de saúde. Sua procura tem-se manifestado sobretudo na região de Lisboa e Vale do Tejo, onde se acentuou no decurso dos últimos três anos.

O presente artigo visa a caracterizar a procura de seguros privados de saúde por sexo, faixa etária e tipo de cobertura, bem como a responder a algumas questões relativas à influência do nível de renda e da existência de doenças crônicas na sua aquisição.

\section{Objetivos}

O primeiro objetivo a atingir com o presente artigo refere-se à existência ou não de um crescimento na população pesquisada com seguro-saúde, por sexo, no Inquérito Nacional de Saúde de 1998/1999 (INS, 1998/1999) em relação ao de 1995/1996 (INS, 1995/1996), primeira pesquisa a coletar esse tipo de informação.

$\mathrm{O}$ artigo pretende, ainda, caracterizar a população com seguro-saúde, no INS 1998/1999, conforme sexo, faixa etária e tipo de cobertura; verificar se há alguma diferenciação na aquisição de seguros-saúde segundo o nível de renda; e averiguar se os portadores de doenças crônicas têm ou não maiores dificuldades para contratar seguros-saúde.

Finalmente, qual a distribuição, em nível regional, da população com seguros privados de saúde no INS 1998/1999 e sua comparação com o INS 1995/1996 é outra questão a que se pretende responder. 


\section{Métodos}

Utilizou-se o INS 1998/1999 para o cálculo da porcentagem de população com seguro privado de saúde, conforme o sexo, a faixa etária e o tipo de cobertura, em relação à população pesquisada em Portugal, na porção continental e em âmbito regional.

O INS 1998/1999 possui uma amostra de 21.808 unidades de alojamento, selecionada de uma amostra-mãe utilizada pelo Instituto Nacional de Estatística (INE), constituída de forma probabilística, por áreas, com base no III Recenseamento da População e Habitação de 1991 (INE, 1994). Numa primeira etapa selecionaram-se as freguesias e, numa segunda fase, procedeu-se à escolha de áreas geográficas bem definidas cartograficamente e com cerca de 300 alojamentos. Nos alojamentos selecionados, todos os indivíduos foram objeto de pesquisa.

A dimensão da amostra assegura a sua representatividade. Prevê-se um erro relativo de amostragem que não ultrapassa os $5 \%$ para apurações anuais. No entanto, dados obtidos na Associação Portuguesa de Seguradores, referentes a uma amostra de 16 companhias de seguros, que representa $90,1 \%$ do ramo doença, evidenciam a existência de cerca de $11 \%$ de pessoas seguradas, contrariamente à amostra do INS 1998/1999 considerada, neste capítulo, de $4,6 \%$.

As perguntas "Tem algum seguro de saúde?" e "Quais os riscos cuja cobertura ele lhe garante?", por faixa etária e por sexo, foram as questões básicas utilizadas neste artigo, incluídas em “Informações gerais de saúde" no INS 1998/ 1999. Não existe diferenciação entre os seguros adquiridos pelas empresas e pelos indivíduos, nem em relação ao titular e dependentes de seguros-saúde.

A primeira questão foi igualmente apurada, em nível regional, a fim de determinar desigualdades de distribuição por sexo, com recurso ao coeficiente de Gini. Procedeu-se à respectiva comparação com coeficientes idênticos calculados para o INS 1995/1996.

Também foram realizadas apurações específicas do INS 1998/1999 em relação à população pesquisada com seguro-saúde, por sexo, grau de escolaridade (ensino básico, secundário e superior) e grupo profissional no qual exerce ou exerceu a profissão. Utilizou-se o grau de escolaridade e a profissão como indicadores indiretos do nível de renda, já que este é dificilmente determinado por meio de pesquisa de opinião.

Procedeu-se igualmente à apuração de segurados com doenças crônicas em relação a três das principais doenças constantes do INS 1998/1999, a saber diabete, asma e bronquite crônica.

\section{Resultados}

A análise comparada do INS 1995/1996 e do INS 1998/1999 demonstra um crescimento, em três anos, respectivamente de $1 \%$ e $0,7 \%$, na porcentagem de população com seguro privado de saúde em relação à população pesquisada dos sexos masculino e feminino, ao passar respectivamente de $4,3 \%$ para $5,3 \%$ e de $3,6 \%$ para $4,3 \%$.

\section{Análise por sexo e por faixa etária}

Vale ressaltar a existência de uma cobertura de $5,3 \%$ na faixa etária de 0 -14 anos, idêntica à dos adultos. A mais elevada porcentagem, 7,3\%, ocorre no sexo masculino, na faixa etária de 3564 anos.

A baixa porcentagem de cobertura dos idosos pode traduzir a dificuldade dessas pessoas em contratar um seguro, devido às suas características de risco e/ou ao fato de terem menos interesse em adquirir seguros privados de saúde por se tratar de um produto novo no mercado.

Christiansen et al. (2001) afirmam que a idade influencia a aquisição do seguro privado de saúde. Segundo eles, a inscrição num seguro privado de saúde aumenta com a idade, até o limite de 60 anos.

Existe uma grande similitude entre a porcentagem de população com seguro-saúde em ambos os sexos, com exceção da faixa etária dos 35-64 anos, na qual se constata a prevalência do sexo masculino. É possível que esse fato tenha relação com uma maior população ativa do sexo masculino e uma maior exposição ao risco em algumas profissões desempenhadas por homens.

\section{Análise por tipo de cobertura}

A análise da população com seguro-saúde por tipo de cobertura evidencia que a maior porcentagem da população pesquisada conta com um seguro que cobre "internação, consultas e exames complementares" (5,3\%), seguida de "internação" (4,8\%) e "internação, consultas, exames complementares e medicamentos" (3,3\%).

A análise por sexo da população, segundo o tipo de cobertura, demonstra uma grande semelhança entre ambos os sexos.

Segundo Propper (2000), a internação, sendo um risco menos freqüente, conduz a que al- 
guns usuários do SNS optem pela aquisição de seguros privados de saúde, ou seja opting-out, específicos para esse serviço.

\section{Análise por nível de renda}

Existe uma clara associação do nível de renda indiretamente traduzido pelo grupo profissional ao qual pertence a população com seguro privado de saúde e seu grau de escolaridade com a procura de seguros-saúde.

Quanto à profissão da população pesquisada com seguro-saúde, os especialistas das profissões intelectuais (respectivamente 19,4\% e $20,1 \%$ nos sexos masculino e feminino), os funcionários superiores da administração pública (respectivamente $14 \%$ e $11,7 \%$ nos sexos masculino e feminino) e os técnicos e pessoal de ní- vel intermediário (respectivamente 14,7\% e $13,3 \%$ nos sexos masculino e feminino) são os que mais adquirem seguros privados de saúde (Tabela 1). Em último lugar situam-se os agricultores (respectivamente $0,8 \%$ e $0,4 \%$ nos sexos masculino e feminino).

Em relação ao grau de escolaridade é, de longe, a população com ensino médio ou superior a que apresenta as porcentagens mais elevadas, respectivamente $21,4 \%$ e $19,5 \%$ nos sexos masculino e feminino. Quanto aos graus de escolaridade restantes, verifica-se uma propensão decrescente para a aquisição de seguros de saúde à medida que o nível de ensino diminui (Tabela 2).

Tabela 1

População pesquisada, com seguro-saúde, por grupo profissional e sexo, no Inquérito

Nacional de Saúde de 1998/1999.

\begin{tabular}{|c|c|c|c|c|c|c|c|c|c|}
\hline \multirow[t]{2}{*}{ Grupos profissionais } & \multicolumn{3}{|c|}{ População pesquisada } & \multicolumn{3}{|c|}{ População com seguro-saúde } & \multicolumn{3}{|c|}{ Percentual } \\
\hline & Homens & Mulheres & Total & Homens & Mulheres & Total & Homens & Mulheres & Total \\
\hline $\begin{array}{l}\text { Funcionários superiores da administração } \\
\text { pública }\end{array}$ & 990 & 513 & 1.503 & 139 & 60 & 199 & 14,0 & 11,7 & 13,2 \\
\hline Especialistas de profissões intelectuais & 708 & 895 & 1.603 & 137 & 180 & 317 & 19,4 & 20,1 & 19,7 \\
\hline Técnicos e pessoal de nível intermediário & 1.274 & 820 & 2.094 & 187 & 109 & 196 & 14,7 & 13,3 & 14,1 \\
\hline Pessoal administrativo e similares & 1.315 & 1.660 & 2.975 & 125 & 150 & 275 & 9,5 & 9,0 & 9,2 \\
\hline Pessoal de serviços, vendedores & 1.473 & 2.419 & 3.892 & 69 & 91 & 160 & 4,7 & 3,8 & 4,1 \\
\hline Agricultores e trabalhadores qualificados & 2.599 & 2.871 & 5.470 & 20 & 11 & 31 & 0,8 & 0,4 & 0,6 \\
\hline Operários, artífices e trabalhadores similares & 5.281 & 1.955 & 7.216 & 134 & 29 & 163 & 2,5 & 1,5 & 2,3 \\
\hline Operadores de instalações e máquinas & 2.310 & 835 & 3.145 & 73 & 21 & 94 & 3,2 & 2,5 & 3,0 \\
\hline Trabalhadores não qualificados & 1.703 & 2.980 & 4.683 & 37 & 41 & 78 & 2,2 & 1,4 & 1,7 \\
\hline Militares de profissão & 89 & 1 & 90 & 5 & 1 & 6 & 5,6 & 100,0 & 6,7 \\
\hline Ignorados/sem profissão & 5.507 & 10.428 & 32.671 & 302 & 398 & 700 & 5,5 & 3,8 & 2,1 \\
\hline
\end{tabular}

Tabela 2

População pesquisada, com seguro-saúde, por grau de escolaridade e sexo, no Inquérito Nacional de Saúde de 1998/1999.

\begin{tabular}{|c|c|c|c|c|c|c|c|c|c|}
\hline \multirow[t]{2}{*}{ Grau de escolaridade } & \multicolumn{3}{|c|}{ População pesquisada } & \multicolumn{3}{|c|}{ População com seguro-saúde } & \multicolumn{3}{|c|}{ Percentual } \\
\hline & Homens & Mulheres & Total & Homens & Mulheres & Total & Homens & Mulheres & Total \\
\hline Só sabe ler e escrever & 1.538 & 3.074 & 4.612 & 5 & 18 & 23 & 0,3 & 0,6 & 0,5 \\
\hline Possui o ensino básico & 12.094 & 10.591 & 22.685 & 501 & 368 & 869 & 4,1 & 3,5 & 3,8 \\
\hline Possui o ensino secundário & 1.521 & 1.471 & 2.992 & 204 & 143 & 347 & 13,4 & 9,7 & 11,6 \\
\hline Possui o ensino médio ou superior & 1.003 & 1.396 & 2.399 & 215 & 272 & 487 & 21,4 & 19,5 & 20,3 \\
\hline
\end{tabular}




\section{Análise por tipo de patologia}

As doenças crônicas afetam a aquisição de seguros privados de saúde. Com efeito, enquanto a porcentagem de população com seguro privado de saúde na população total pesquisada é de $4,8 \%$, verificam-se porcentagens inferiores em relação às principais doenças crônicas incluídas no INS 1998/1999, designadamente em relação à asma $(3,4 \%)$, à bronquite crônica $(2,5 \%)$ e, principalmente, à diabete $(1,6 \%)$ (Tabela 3$)$.

\section{Desigualdades regionais}

A análise regional do INS 1998/1999 evidencia que a região de Lisboa e Vale do Tejo é a que apresenta uma porcentagem superior à média da porção continental de Portugal, com valores respectivamente de $8,8 \%$ e $7,0 \%$ nos sexos masculino e feminino. Segue-se a região Centro, com porcentagens de $4,7 \%$ e $3,5 \%$, e a região do Alentejo, com valores de $4,4 \%$ e $4 \%$ (Tabela 4 ). A região do Algarve apresenta o valor mínimo, tanto no sexo masculino como no feminino.

O cálculo do coeficiente de Gini em relação às desigualdades regionais do seguro privado de saúde demonstra que aumentou a desigualdade inter-regional em ambos os sexos (Tabela 5). O coeficiente de Gini, que era de 0,1549 no INS 1995/1996, no sexo masculino, passou para 0,2356 no INS 1998/1999, aproximando-se do valor no sexo feminino, que era de 0,2285 e é agora de 0,2775. Esse aumento deve-se principalmente a um maior crescimento na aquisição de seguros privados de saúde, em ambos os sexos, na região de Lisboa e Vale do Tejo, seguida da região Centro, relativamente às outras regiões.

\section{Conclusões}

A análise comparada do INS 1995/1996 e INS 1998/1999 evidencia um crescimento em três anos na porcentagem de população com seguro privado de saúde, tanto no sexo masculino como feminino.

Existe grande similitude entre a porcentagem de população com seguro-saúde, em ambos os sexos, com exceção da faixa etária dos 35-64 anos, na qual a população com segurosaúde é mais numerosa no sexo masculino.

Existe uma clara associação do nível de ren$\mathrm{da}$ - indiretamente traduzido pelo grupo profissional ao qual pertence a população com seguro privado de saúde e seu grau de escolaridade - com a procura de seguros-saúde. Os especialistas das profissões intelectuais são os que mais adquirem esses seguros.

Quanto ao grau de escolaridade, a população com ensino médio ou superior é a que apresenta as porcentagens mais elevadas de aquisição de seguros privados de saúde.

As doenças crônicas afetam igualmente o acesso a esse tipo de seguro. Enquanto a por-

Tabela 3

População pesquisada, com seguro-saúde, por principal doença crônica, no Inquérito Nacional de Saúde de 1998/1999.

\begin{tabular}{lccc}
\hline Doença crônica & $\begin{array}{c}\text { População } \\
\text { pesquisada }\end{array}$ & $\begin{array}{c}\text { População com } \\
\text { seguro-saúde }\end{array}$ & $\%$ \\
\hline Diabete & 2.580 & 42 & 1,6 \\
Asma & 2.882 & 97 & 3,4 \\
Bronquite crônica & 1.380 & 35 & 2,5 \\
\hline
\end{tabular}

Tabela 4

Porcentagem da população pesquisada que declarou ter seguro-saúde, nos Inquéritos

Nacionais de Saúde de 1995/1996 e 1998/1999, por região.

\begin{tabular}{|c|c|c|c|c|c|c|c|c|}
\hline \multirow[t]{3}{*}{ Regiões } & \multicolumn{4}{|c|}{ INS 1995/1996 } & \multicolumn{4}{|c|}{ INS 1998/1999 } \\
\hline & \multicolumn{2}{|c|}{ Valor } & \multicolumn{2}{|c|}{$\%$} & \multicolumn{2}{|c|}{ Valor } & \multicolumn{2}{|c|}{$\%$} \\
\hline & Homens & Mulheres & Homens & Mulheres & Homens & Mulheres & Homens & Mulheres \\
\hline Norte & 296 & 231 & 4,3 & 3,0 & 266 & 241 & 3,8 & 3,1 \\
\hline Centro & 194 & 153 & 4,1 & 2,9 & 216 & 177 & 4,7 & 3,5 \\
\hline Lisboa & 443 & 397 & 6,8 & 5,6 & 524 & 476 & 8,8 & 7,0 \\
\hline Alentejo & 117 & 83 & 4,6 & 2,6 & 127 & 122 & 4,4 & 4,0 \\
\hline Algarve & 100 & 75 & 3,9 & 2,8 & 95 & 75 & 3,4 & 2,6 \\
\hline Total & 1.150 & 939 & 4,3 & 3,6 & 1.228 & 1.091 & 5,3 & 4,3 \\
\hline
\end{tabular}


Tabela 5

Coeficientes de Gini da população pesquisada, nos Inquéritos Nacionais de Saúde de 1995/1996 e de 1998/1999, que declarou ter seguro-saúde, por região.

\begin{tabular}{|c|c|c|}
\hline \multirow{2}{*}{ Seguro-saúde } & \multicolumn{2}{|c|}{ Coeficientes de Gini ${ }^{1}$} \\
\hline & Homens & Mulheres \\
\hline INS 1995/1996 & 0,1549 & 0,2285 \\
\hline INS 1998/1999 & 0,2356 & 0,2775 \\
\hline \multicolumn{3}{|c|}{$\begin{array}{l}1 \text { O coeficiente de Gini varia entre } 0 \text { e } 1 \text {, no qual } 1 \text { corresponde ao valor com mais } \\
\text { elevada desigualdade. Obtém-se de acordo com a seguinte fórmula: }\end{array}$} \\
\hline 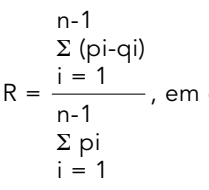 & isada e qi & medir. \\
\hline
\end{tabular}

centagem de população com seguro privado de saúde na população total pesquisada é de $4,8 \%$, verificam-se porcentagens inferiores em relação às principais doenças crônicas incluídas no INS 1998/1999, designadamente em relação à asma, à bronquite crônica e, principalmente, à diabete.

Em relação às desigualdades regionais, o cálculo do coeficiente de Gini demonstra que aumentou a desigualdade inter-regional em relação ao INS 1995/1996, para ambos os sexos. Esse aumento deve-se, sobretudo, a um maior crescimento relativo na aquisição de seguros privados de saúde na região de Lisboa e Vale do Tejo, tanto no sexo masculino como no feminino.

\section{Referências}

CHRISTIANSEN, T.; LAURIDSEN, J. \& KAMPER-JOERGENSEN, F., 2001. Demand for Private Health Insurance and Demand for Health Care by Privately Insured in Denmark - With a Specific View to Equity. Odense: Department of Public Health, University of Southern Denmark. (mimeo.)

FELDMAN, R.; ESCRIBANO, C. \& PELLISE, L., 1998. The role of government in health insurance markets with adverse selection. Health Economics, 7:659-670.

GIRALDES, M. R., no prelo. Desigualdades regionais nos subsistemas de saúde de Portugal. Análise Social.

INS (Inquérito Nacional de Saúde), 1995/1996. Continente. Dados Gerais. Lisboa: Departamento de Estudos e Planejamento da Saúde.
INS (Inquérito Nacional de Saúde), 1998/1999. Continente. Dados Gerais. Lisboa: INS.

MARCHAND, M. \& SCHROYEN, F., 2001. Markets for Public and Private Health Care: Redistribution Arguments for a Mixed System. Discussion Paper 23. Louvain: Center for Operations Research and Econometrics, Université Catholique de Louvain.

NEUDECK, W. \& PODEZECK, K., 1996. Adverse selection and regulation in health insurance markets. Journal of Health Economics, 15:387-408.

PROPPER, C., 2000. The demand for private health care in the UK. Journal of Health Economics, 19: 855-876.

Recebido em 4 de fevereiro de 2002

Versão final reapresentada em 21 de junho de 2002 Aprovado em 2 de setembro de 2002 